\title{
Ophthalmological features and treatments in five cases of Waardenburg syndrome
}

\author{
YAN LIU* ${ }^{*}$,HUI PAN ${ }^{*}$, JING WANG, QINKE YAO, MING LIN, BO MA and JIN LI \\ Department of Ophthalmology, Ninth People's Hospital, \\ Shanghai JiaoTong University School of Medicine, Shanghai 200011, P.R. China
}

Received September 6, 2019; Accepted June 19, 2020

DOI: $10.3892 /$ etm.2020.9035

\begin{abstract}
The present study aimed to investigate the ocular characteristics and treatment of Waardenburg syndrome (WS). A total of five patients with Waardenburg syndrome from our hospital, aged between 1 and 8 years, were included in the present study. The clinical data of these patients were analyzed, and the ocular manifestations and treatments were described. The general manifestations included hearing loss (1/5), broad high nasal root (2/5) and hypoplasia of alae nasi (2/5). Ophthalmological evaluations revealed ptosis $(1 / 5)$, strabismus $1(1 / 5)$, synophrys $(2 / 5)$, telecanthus $(5 / 5)$, iris hypopigmentation $(5 / 5)$, high intraocular pressure $(1 / 5)$ and choroidal hypopigmentation $(1 / 5)$. For patients with characteristic external eye abnormalities, including ptosis, ocular plastic surgery was performed. For patients with only symptoms of iris heterochromia, no special treatment was required. The findings from the present study suggest that patients with WS may have several characteristic ocular manifestations. Abnormalities in the eyelid can be corrected by ocular plastic surgery, which is beneficial to children's physical and mental development.
\end{abstract}

\section{Introduction}

Waardenburg syndrome (WS) is a rare genetic disorder characterized by various degrees of deafness, abnormal pigmentation and minor defects in structures arising from neural crest. WS was first introduced by the ophthalmologist Petrus J Waardenburg in 1947 and was described in further detail in $1951(1,2)$. Overall, the syndrome affects 1 in 42,000 people

Correspondence to: $\mathrm{Dr}$ Bo Ma or $\mathrm{Dr} \mathrm{Jin} \mathrm{Li}$, Department of Ophthalmology, Ninth People's Hospital, Shanghai JiaoTong University School of Medicine, 639 Zhizaoju Road, Shanghai 200011, P.R. China

E-mail: boma1983@hotmail.com

E-mail: lijinabcd@sina.cn

*Contributed equally

Key words: Waardenburg syndrome, ocular manifestations, ptosis, ocular plastic surgery in the population (1). WS is caused by mutations of several genes that affect the division and migration of neural crest cells during embryonic development. Pathogenic genes associated with WS include PAX3, MITF, SNAI2, EDN3, EDNRB and SOX10 (2,3). The clinical characteristics of the syndrome include lateral displacement of the eye's medial canthi, a hyperplastic broad high nasal root, hyperplasia of the medial portions of the eyebrows, partial or total heterochromia iridum, congenital deafness or partial (unilateral) deafness and circumscribed albinism of the frontal head hair (white forelock) (1). Currently, there are four different types of WS according to various clinical characteristics. WS type 1 is characterized by dystopia canthorum, congenital sensorineural hearing loss, pigmentary disturbances of iris and hair hypopigmentation (2). WS type 1 and WS type 2 are distinguished by the presence or absence of dystopia canthorum, respectively. WS type 3 (Klein-Waardenburg syndrome) is similar to type 1 with additional musculoskeletal abnormalities. WS type 4 (Shah-Waardenburg syndrome or Waardenburg-Hirschsprung disease) is characterized by the presence of an aganglionic megacolon (3). The highly variable presentations of WS make it difficult to reach a definitive diagnosis. Studies reporting ocular manifestations are limited. Müllner-Eidenböck et al (4) presented Turkish family members with WS type 2 who presented with a fundus photo with ipsilateral connections between the iris and fundus. Cortés-González et al (5) suggested that posterior microphthalmos may be associated with WS type 2A. Previous studies have also reported that patients with WS may suffer from additional open-angle glaucoma or branch retinal vein occlusion $(6,7)$. Furthermore, Meire et al (8) reported a patient with WS who presented with Marcus Gunn ptosis with jaw-winking. Despite numerous findings connecting WS syndrome and intraocular abnormalities, little is known regarding the abnormal configuration of the eye and its comorbidities in WS. Therefore, the present article focused on the external ocular manifestations in five patients diagnosed with WS, and suggested potential treatments to improve the ocular appearance of these patients.

\section{Materials and methods}

The present study followed the principles of the Declaration of Helsinki and was approved by The Ethics Committee of the Ninth People's Hospital, Shanghai Jiao Tong University School 
of Medicine. Informed consent for publication of the images was provided by the guardians of the patients. A total of five Chinese patients with a clinical diagnosis of WS from the same age group, examined in the Department of Ophthalmology, Ninth People's Hospital, Shanghai Jiao Tong University School of Medicine (Shanghai, China) between March 2014 and July 2018, were included in the present study. The criteria for diagnosis of WS were based on the Waardenburg Consortium, indicating that affected individuals must demonstrate at least 2 major criteria, or 1 major criterion plus 2 minor criteria (Table I) $(2,9,10)$.

Data collected from patients included age, sex, family history of WS, hair color, skin signs, hearing (confirmed by audiology testing) and characteristic facial features (eyebrow shape, nasal profile and nasal alae). No patients presented with systemic diseases. Each patient underwent visual acuity, slit lamp, fundus and external ocular examination. The collected data involved color analysis of the iris and fundus images by anterior segment and fundus photography, intraocular pressure, retinal nerve fiber layer thickness and cup/disc ratio. External ocular examination includes eyelid measurements of palpebral fissure width (FW), interpupillary distance, inner and outer canthal distance, upper eyelid margin reflex distance (the distance from the central corneal light reflection to the upper lid margin distance; MRD1) $(11,12)$ and levator muscle function (LMF).

Surgical correction of ptosis was performed by one surgeon (JL). The different plastic procedures (levator resection or frontalis suspension) depended on the degree of ptosis and inner canthus forms (13-15). Patients with glaucoma were treated with anti-glaucoma medications (Carteolol hydrochloride $1 \%$ twice a day) (16). For patients with iris heterochromia only, follow-up lasted for 2-3 years, including visual acuity, intraocular pressure and external ocular examinations.

\section{Results}

A total of five Chinese patients with WS (four male and one female) were included in the present study. The mean age at presentation was 3.8 years (median age, 3.5 years; age range, 1-8 years). All patients met the diagnostic criteria for WS type 1 (Table I). No patient had muscle contractures (WS type 3) or Hirschsprung disease (WS type 4). As listed in Table II, the general manifestations included hearing loss (1/5), broad high nasal root (1/5) and hypoplasia of alae nasi (1/5). Ophthalmological evaluations demonstrated ptosis $(1 / 5)$, strabismus $(1 / 5)$, synophrys $(2 / 5)$, dystopia canthorum $(\mathrm{W}>1.95)(5 / 5)$, iris hypopigmentation $(5 / 5)$, high intraocular pressure (1/5) and choroidal hypopigmentation (1/5). The ophthalmological manifestations are presented in Table III.

Case 1. A 3-year and 6-month-old male child presented with ptosis in his right eye (Fig. 1). Prior to examination, the patient had a history of congenital bilateral hearing loss and was treated by cochlear implantation for 2 years. The patient exhibited complete iris hypopigmentation (pale blue eyes) bilaterally. He did not have any neurological defect or skin abnormality, and had no family history of WS. The uncorrected distance visual acuity (UDVA) was 20/80 in the right eye and was $20 / 50$ in the left eye. Cycloplegic refraction for the right eye [oculus dexter (OD)] was: +3.25 diopters sphere (DS)/-0.75 diopters cylinder (DC) $\times 160^{\circ}$, with a corrected distance visual acuity (CDVA) of 20/50. Cycloplegic refraction for the left eye [oculus sinister (OS)] was: $+2.75 \mathrm{DS} /-0.75 \mathrm{DC} \times 150^{\circ}$, with CDVA of 20/30. The FW was $17 \mathrm{~mm}$ in the right eye and was $18 \mathrm{~mm}$ in the left eye. The MRD1 was 0 in the right eye and $3 \mathrm{~mm}$ in the left eye. The LMF was $2 \mathrm{~mm}$ in the right eye and $9 \mathrm{~mm}$ in the left eye. For the development of vision, ptosis of the patient was surgically corrected by levator resection. The patient underwent timely treatment of amblyopia in order to improve visual function (17). Current treatment for amblyopia mainly includes optical correction, patching, pharmacological treatment, optical treatment, Bangerter filters and surgery (17). After 3 years of postoperative amblyopia therapy the patient's visual function was well developed. The UDVA was 20/30 in the right eye and 20/20 in the left eye. Cycloplegic refraction was OD: +2.75 DS/-0.75 DC x160', with CDVA of 20/20, and OS: $+1.50 \mathrm{DS} /-1.25$ DC $\times 160^{\circ}$, with CDVA of $20 / 20$. This patient underwent levator resection in hospital, and him and his parents underwent genetic testing. Complete sequence analysis of PAX3, MITF and SOX10 genes was performed. The results demonstrated that no pathogenic mutations were detected.

Case 2. An 8-year-old male child presented with strabismus in his left eye (Fig. 2). Ophthalmological examination demonstrated complete iris hypopigmentation (pale blue eye) unilaterally. There was an increased difference between the medial canthus of the eyes and broad nasal root. The inner canthal distance was $55 \mathrm{~mm}$. The FW was $19 \mathrm{~mm}$, the MRD1 was $3 \mathrm{~mm}$ and the LMF was $10 \mathrm{~mm}$ in both eyes. No hearing loss, neurological defects or skin abnormalities were reported in this case. The binocular vision was 20/20 and there was a post-equatorial hypopigmented choroid with scattered pigmented spots in the right eye compared with the left one. Examination showed enlarged cup to disc ratio (C/D) in both eyes [oculus uterque $[(\mathrm{OU})(\mathrm{OU} \mathrm{C} / \mathrm{D}=0.6)]$ and high intraocular pressure $(\mathrm{OD}=35 \mathrm{mmHg}, \mathrm{OS}=33 \mathrm{mmHg}$ ), but RNFL thickness was within the normal range, suggesting early onset of elevated intraocular pressure. The type of glaucoma was open angle glaucoma. The peripheral anterior chamber depth was $>1 / 3$ of the peripheral corneal thickness in slit-lamp examination and no peripheral anterior synechia was seen. The patient was treated with anti-glaucoma eye drops (Carteolol hydrochloride $1 \%$ twice a day) and the intraocular pressure was well controlled at the 3-year follow-up. There was a post-equatorial hypopigmented choroid with scattered pigmented spots in the right fundus photo. The enlarged inner canthal distance did not affect the patient's visual function, and no plastic surgery was needed.

Cases 3-5. Cases 3-5 presented with the initial complaint of abnormal color of the iris (Fig. 3). The children in case 3 (a 1-year and 5-month-old boy) and case 4 (a 1-year-old boy) were too young to perform a visual examination; the eye examination therefore only assessed eyesight, which was followed up regularly. The MRD1 was $3 \mathrm{~mm}$ and the LMF was $10 \mathrm{~mm}$ in both eyes in case 3 . In case 4 , the MRD1 was $4 \mathrm{~mm}$ and the LMF was $10 \mathrm{~mm}$ in both eyes. Furthermore, 
Table I. Waardenburg Consortium diagnostic criteria for Type 1.

Major criteria

Minor criteria

Congenital sensorineural hearing loss

Pigmentary disturbance of iris

Pigmentary disturbance of hair (white forelock)

Dystopia canthorum, $\mathrm{W}$ index $>1.95$

Affected first degree relative
Congenital hypopigmentation of the skin

Synophrys (eyebrows that meet)

Broad high nasal root

Hypoplasia of alae nasi

Premature greying of hair

To calculate the $\mathrm{W}$ index (in $\mathrm{mm}$ ): i) Measure inner canthal distance (a), interpupillary distance (b), outer canthal distance (c); ii) Calculate $X=(2 a-0.2119 c-3.909) / c ; Y=(2 a-0.2479 b-3.909) / b$; and iii) Calculate $W=X+Y+a / b$.

during examination of case 5 (A 5-year-old girl), the patient was diagnosed with a slight photophobia, although no intraocular defect was found except for iris morphology. The visual acuity was 20/20 in both eyes. The MRD1 was $4 \mathrm{~mm}$ and the LMF was $11 \mathrm{~mm}$ in both eyes. For patients with symptoms of iris heterochromia only, no special treatment was required and colored contact lenses could be worn.

\section{Discussion}

WS is characterized by a variety of typical clinical manifestations caused by pigmentation disorders. Pathogenic genes related to WS include PAX3, MITF, SNAI2, EDN3, EDNRB and SOX10 (2,3). In the present study, the patient in case 1 underwent levator resection due to ptosis in hospital; therefore, the patient and their parents underwent genetic testing. Complete sequence analysis of the PAX3, MITF and SOX10 genes was performed. The results demonstrated that no pathogenic mutations were detected. The other four children were examined at the outpatient clinic and diagnosed with WS type 1. At the time of the study, there was no special treatment, and their parents declined genetic testing.

The clinical manifestations of pigment synthesis disorders include premature whitening of the hair, hypopigmentation of the skin and heterochromia of the iris, accompanied by various degrees of sensorineural hearing loss. Patients may also have other systematic signs, such as dystopia canthorum, dysplastic skeletal muscles of the extremities, Hirschsprung disease and abnormalities of the intestinal nervous system (18). According to Farrer's 1992 WS diagnostic criteria (9), all cases in the present study were classified as WS type 1. Certain signs, including ptosis, synophrys, telecanthus, iris hypopigmentation, choroidal hypopigmentation, high intraocular pressure and strabismus were identified. For WS, the ocular abnormal manifestations are diverse. Subsequently, the eyes of patients with WS must be examined carefully to rule out eye problems. If there are any problems, proper intervention and treatment are necessary.

Ptosis, which refers to abnormal drooping of the upper lid due to partial or total loss of levator function (11), is one of the most common congenital abnormalities in children, although it is a rare characteristic in WS. Clinical features include continuous limitations in the visual field and, and in some severe cases, amblyopia. Patients may need to raise their eyebrows or lift their eyelid with a finger (19). The specific facial features, including increased forehead lines, raised eyebrows and looking upwards, not only affect appearance but also affect the patient's psychological development to varying degrees. Surgery is an effective method of correcting ptosis. Ptosis has been reported in the literature regarding WS, but it has not been described in detail, nor have specific treatments been described. Chen et al (3) and Tamayo et al (20) reported cases of ptosis in WS; however, they did not discuss treatments. Meire et al (8) hypothesized that the occurrence of Marcus Gunn ptosis in a patient with WS may suggest a lesion in the sympathetic pathway.

The correct time for surgery in WS-associated ptosis is not well established. Since the patient can prevent the interference of the upper eyelid when looking up or looking down, less amblyopia, strabismus or refractive errors occur. Therefore, for mild to moderate ptosis, surgery is considered to be better at 3-6 years of age. For severe cases, early intervention is suggested to protect their visual development and prevent amblyopia. In case 1 of the present study, the levator muscle strength of the patient was $2 \mathrm{~mm}$, which was a severe ptosis that met the surgical correction criteria. The operation method was levator resection. Following the operation, the ptosis of the patient was well corrected. After 3 years of postoperative amblyopia training, the patient's visual function was well developed. The UDVA was 20/30 in the right eye and $20 / 20$ in the left eye. Cycloplegic refraction was OD: $+2.75 \mathrm{DS} /-0.75 \mathrm{DC} \times 160^{\circ}$ with CDVA of $20 / 20$, and OS: $+1.50 \mathrm{DS} /-1.25$ DC $\times 160^{\circ}$ with CDVA of $20 / 20$. No amblyopia was observed in this patient. Previous studies have reported that children with amblyopia due to astigmatic anisometropia or deprivation could benefit from early surgical correction $(21,22)$.

Kadoi et al (7) reported a case of WS with hypopigmented fundi, branch retinal vein occlusion and high intraocular pressure. Nork et al (23) and Gupta and Aggarwal (6) reported cases of WS with bilateral glaucoma. Although glaucoma has not been considered as an associated characteristic of WS, a possible mechanism could be that ocular melanocytes may be derived from the neural crest and that a defect in pigmentation may therefore lead to developmental abnormalities in these structures. As discussed in the literature, the iridocorneal angle structures are largely of neural crest origin, and the predisposition to glaucoma should be investigated with a larger number of WS cases, which may support the association 

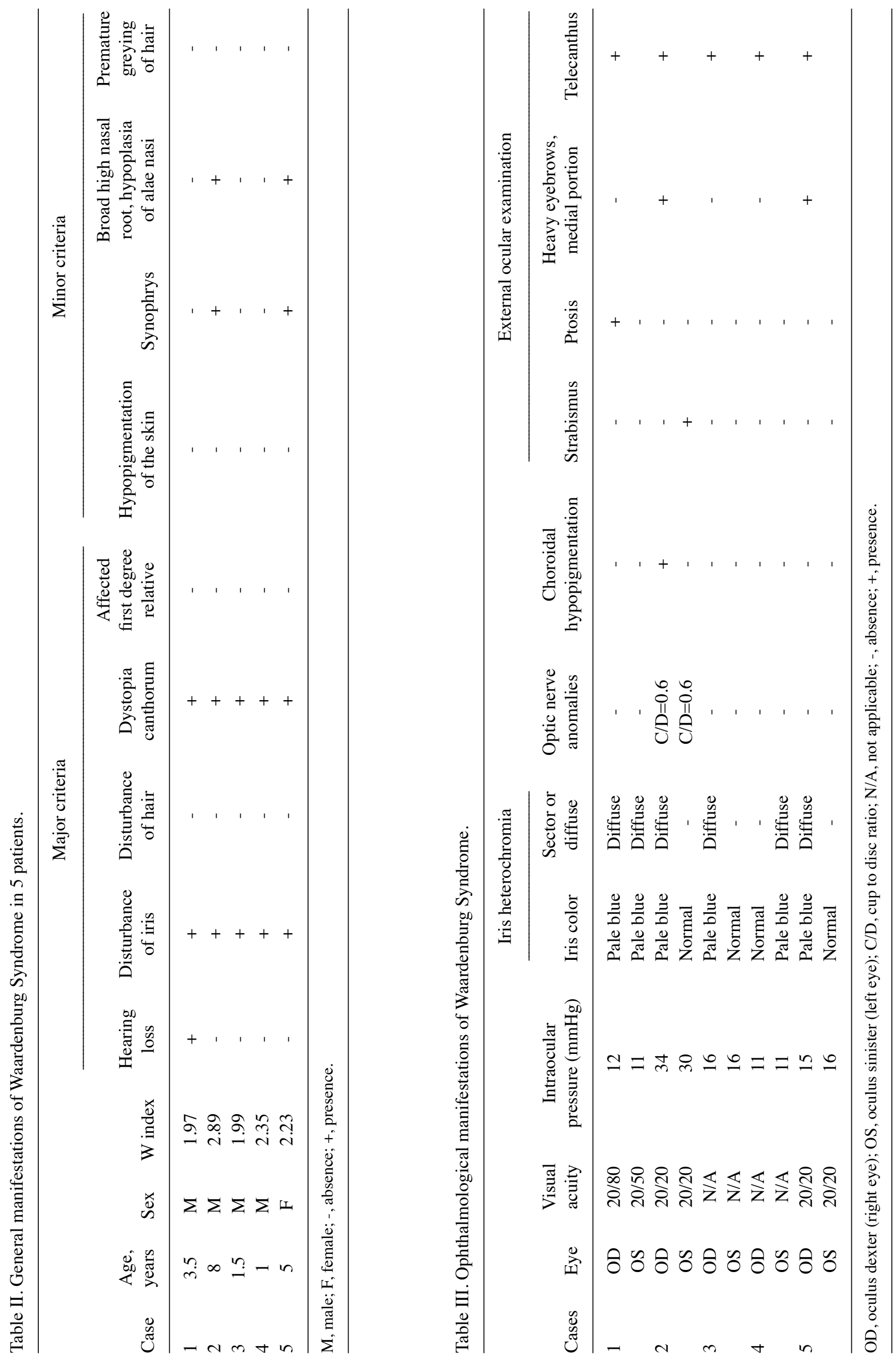

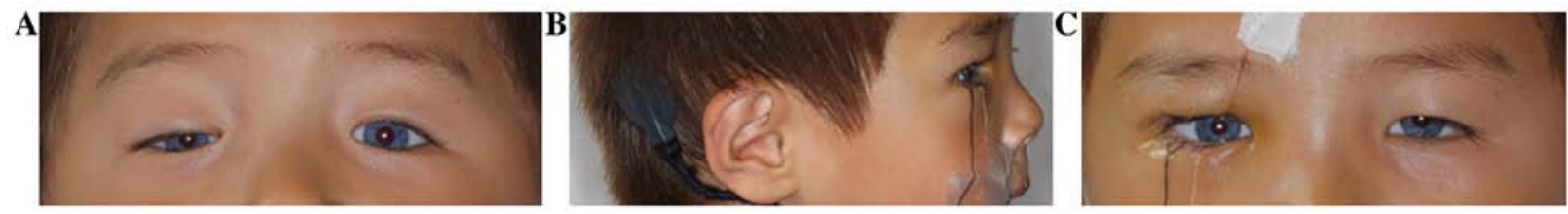

Figure 1. (A) A 3-year-old and 6-month-old boy presented with ptosis in his right eye and complete iris hypopigmentation bilaterally. (B) The patient was treated by cochlear implantation. (C) Postoperative photograph demonstrated improved margin reflex distance 1.
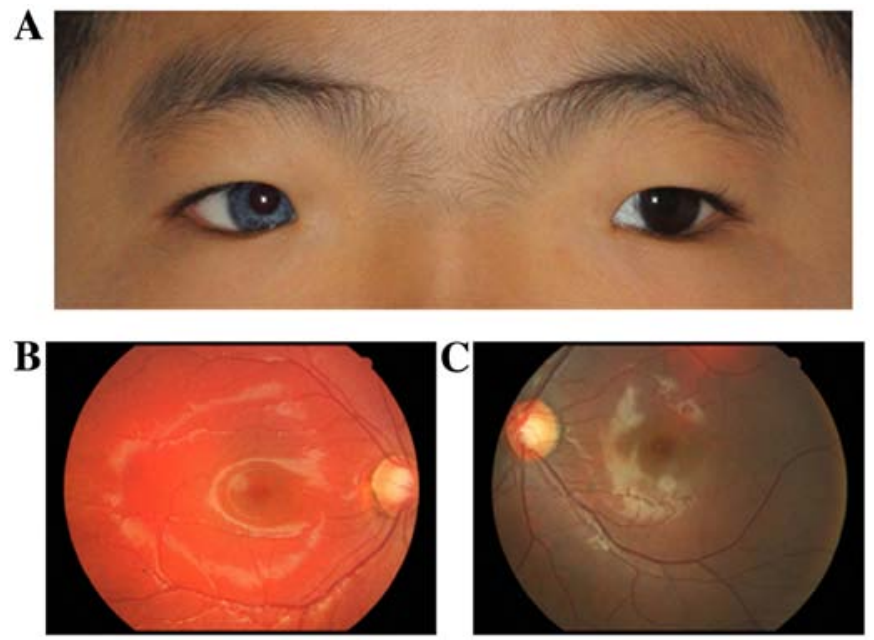

Figure 2. (A) An 8-year-old male child with telecanthus, synophrys, strabismus and complete iris hypopigmentation unilaterally. (B) In the funduscopic test, there was a post-equatorial hypopigmented choroid with scattered pigmented spots in the right eye compared with the left one. (C) Fundus photograph of the left eye.
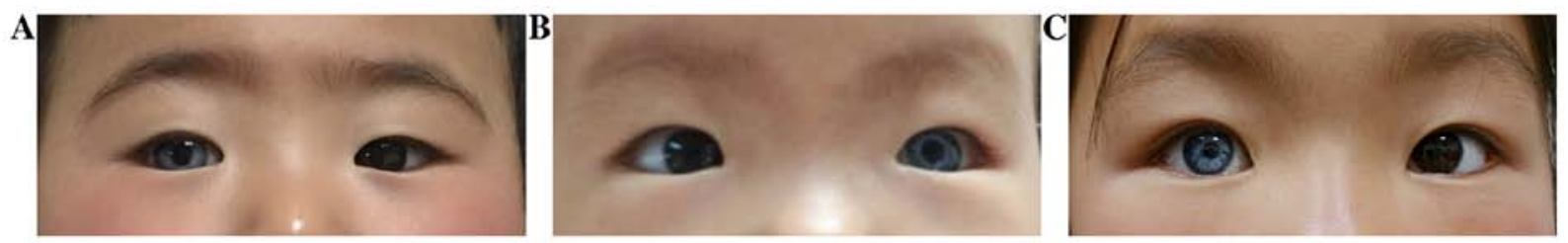

Figure 3. (A) A 1-year and 6-month-old boy (case 3) with iris hypopigmentation in his right eye. (B) A 1-year old male patient i (case 4) with left iris heterochromia. (C) A 5-year-old girl (case 5) presented with an abnormal color of the right eye, synophrys and hypoplasia of alae nasi.

of glaucoma with WS (6). In the case 2 of the present study, the patient had high intraocular pressure and enlarged cup to disc ratio. This patient was treated with anti-glaucoma eye drops and follow-up observation was needed regularly. This finding suggested that examination of intraocular pressure, optic disc ratio and RNFL measurement for patients with WS may be necessary.

The characteristic ocular abnormality of WS is abnormal pigmentation of the iris. Iris hypopigmentation occurred in all 5 patients from the present study. Changes in iris pigmentation in WS included the following: Complete heterochromia (different colors in both eyes), partial heterochromia (segmented blue) and stunted bright blue iris (24). In the present study, all 5 patients presented with complete heterochromia and no partial heterochromia was observed. Patients with heterochromia of the iris in WS may need to use sunglasses to protect the intraocular structures of the eye against excess radiation from the sun's rays. Colored contact lenses can be used for cosmetic reasons.
In the present study, several abnormal characteristics of the eyes were reported, including synophrys, epicanthal folds, telecanthus, ptosis and strabismus. Not only the external abnormalities, but also the intraocular defects of patients with WS should be carefully examined in clinic. Abnormalities in the external eye, such as ptosis, epicanthal folds or telecanthus, can be corrected by ocular plastic surgery, which is beneficial to the physical development of child.

\section{Acknowledgements}

Not applicable.

\section{Funding}

This work was supported by the National Natural Science Foundation of China (grant nos. 81870688 and 81970834) and the Science and Technology Commission of Shanghai (grant no. 17DZ2260100). 


\section{Availability of data and materials}

The datasets used and/or analyzed during the current study are available from the corresponding author on reasonable request.

\section{Authors' contributions}

YL and HP obtained the data and wrote the manuscript. JW, QY and ML analyzed the datasets. BM and LJ designed the study and critically revised the manuscript. All authors read and approved the final manuscript.

\section{Ethics approval and consent to participate}

This study was approved by the Ethics Committee of the Ninth People's Hospital, Shanghai Jiao Tong University School of Medicine (Shanghai, China).

\section{Patient consent for publication}

Informed consent for publication of the images was provided by the guardians of the patients

\section{Competing interests}

The authors declare that they have no competing interests.

\section{References}

1. Waardenburg PJ: A new syndrome combining developmental anomalies of the eyelids, eyebrows and nose root with pigmentary defects of the iris and head hair and with congenital deafness. Am J Hum Genet 3: 195-253, 1951.

2. Read AP and Newton VE: Waardenburg syndrome. J Med Genet 34: 656-665, 1997.

3. Chen H, Jiang L, Xie Z, Mei L, He C, Hu Z, Xia K and Feng Y: Novel mutations of PAX3, MITF, and SOX10 genes in Chinese patients with type I or type II Waardenburg syndrome. Biochem Biophys Res Commun 397: 70-74, 2010.

4. Müllner-Eidenböck A, Moser E, Frisch H and Read AP: Waardenburg syndrome type 2 in a Turkish family: Implications for the importance of the pattern of fundus pigmentation. Br J Ophthalmol 85: 1384-1386, 2001

5. Cortés-González V, Zenteno JC, Guzmán-Sánchez M, Giordano-Herrera V, Guadarrama-Vallejo D, Ruíz-Quintero N and Villanueva-Mendoza C: Tietz/Waardenburg type 2A syndrome associated with posterior microphthalmos in two unrelated patients with novel MITF gene mutations. Am J Med Genet A 170: 3294-3297, 2016.

6. Gupta V and Aggarwal HC: Open angle glaucoma as a manifestation of Waardenburg's syndrome. Indian J Ophthalmol 48: 49-50, 2000.

7. Kadoi C, Hayasaka S and Yamamoto S: Branch retinal vein occlusion in a patient with Waardenburg syndrome. Ophthalmologica 210: 354-357, 1996.

8. Meire F, Standaert L, De Laey JJ and Zeng LH: Waardenburg syndrome, Hirschsprung megacolon, and Marcus Gunn ptosis Am J Med Genet 27: 683-686, 1987.
9. Farrer LA, Grundfast KM, Amos J, Arnos KS, Asher JH Jr Beighton P, Diehl SR, Fex J, Foy C, Friedman TB, et al: Waardenburg syndrome (WS) type I is caused by defects at multiple loci, one of which is near ALPP on chromosome 2: First report of the WS consortium. Am J Hum Genet 50: 902-913, 1992.

10. Shields CL, Nickerson SJ, Al-Dahmash S and Shields JA: Waardenburg syndrome: Iris and choroidal hypopigmentation: Findings on anterior and posterior segment imaging. JAMA Ophthalmol 131: 1167-1173, 2013.

11. Li J, Lin M, Zhou H, Jia R and Fan X: Double-eyelid blepharoplasty incorporating blepharoptosis surgery for 'latent' aponeurotic ptosis. J Plast Reconstr Aesthet Surg 64: 993-999, 2011.

12. Quaranta-Leoni FM, Sposato S, Leonardi A, Iacoviello L and Costanzo S: Timing of surgical correction for the treatment of unilateral congenital ptosis: Effects on cosmetic and functional results. Orbit 36: 382-387, 2017.

13. Clauser L, Tieghi R and Galie M: Palpebral ptosis: Clinical classification, differential diagnosis, and surgical guidelines: An overview. J Craniofac Surg 17: 246-254, 2006.

14. Gazzola R, Piozzi E, Vaienti L and Wilhelm Baruffaldi Preis F: Therapeutic algorithm for congenital ptosis repair with levator resection and frontalis suspension: Results and literature review. Semin Ophthalmol 33: 454-460, 2018.

15. Dave TV, Sharma P, Nayak A, Moharana R and Naik MN: Outcomes of frontalis sling versus levator resection in patients with monocular elevation deficiency associated ptosis. Ophthalmic Plast Reconstr Surg 35: 251-255, 2019.

16. Chrisp P and Sorkin EM: Ocular carteolol. A review of its pharmacological properties, and therapeutic use in glaucoma and ocular hypertension. Drugs Aging 2: 58-77, 1992.

17. Wallace DK, Repka MX, Lee KA, Melia M, Christiansen SP, Morse CL and Sprunger DT; American Academy of Pediatric Ophthalmology/Strabismus Preferred Practice Pattern Pediatric Ophthalmology Panel: Amblyopia preferred practice pattern ${ }^{\circledR}$. Ophthalmology 125: P105-P142, 2018.

18. Liu XZ, Newton VE and Read AP: Waardenburg syndrome type II: Phenotypic findings and diagnostic criteria. Am J Med Genet 55: 95-100, 1995.

19. Cahill KV, Bradley EA, Meyer DR, Custer PL, Holck DE, Marcet MM and Mawn LA: Functional indications for upper eyelid ptosis and blepharoplasty surgery: A report by the American Academy of Ophthalmology. Ophthalmology 118: 2510-2517, 2011

20. Tamayo ML, Gelvez N, Rodriguez M, Florez S, Varon C, Medina D and Bernal JE: Screening program for Waardenburg syndrome in Colombia: Clinical definition and phenotypic variability. Am J Med Genet A 146A: 1026-1031,2008.

21. Akal A, Göncü T, Boyaci N and Yilmaz ÖF: Anisometropic amblyopia in a case of type 2 Waardenburg syndrome. BMJ Case Rep 2013: bcr2013201140, 2013.

22. SooHoo JR, Davies BW, Allard FD and Durairaj VD: Congenital ptosis. Surv Ophthalmol 59: 483-492, 2014.

23. Nork TM, Shihab ZM, Young RS and Price J: Pigment distribution in Waardenburg's syndrome: A new hypothesis. Graefes Arch Clin Exp Ophthalmol 224: 487-492, 1986.

24. Ohno N, Kiyosawa M, Mori H, Wang WF, Takase H and Mochizuki M: Clinical findings in Japanese patients with Waardenburg syndrome type 2. Jpn J Ophthalmol 47: 77-84, 2003.

This work is licensed under a Creative Commons Attribution-NonCommercial-NoDerivatives 4.0 International (CC BY-NC-ND 4.0) License. 